
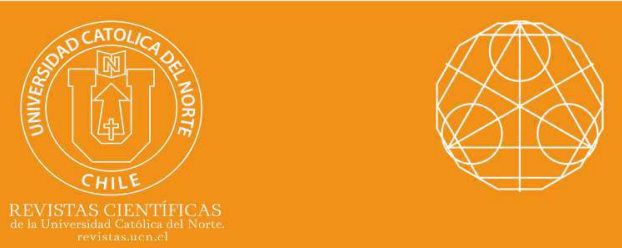

\title{
On the pseudospectrum preservers
}

\author{
Mustapha Ech-Chérif El Kettani ${ }^{1}$ () orcid.org/0000-0003-0072-4415 \\ Aziz Lahssaini ${ }^{2}$ 이 orcid.org/0000-0001-8114-170X \\ University Sidi Mohammed Ben Abdellah, Dept of Mathematics, LaSMA Laboratory, Fes, Morocco. \\ 1 mostapha.echcherifelkettani@usmba.ac.ma; ${ }^{2}$ aziz.lahssaini@usmba.ac.ma
}

\section{Abstract:}

Let $X$ and $Y$ be two complex Banach spaces, and let $B(X)$ denotes the algebra of all bounded linear operators on $X$. We characterize additive maps from $B(X)$ onto $B(Y)$ compressing the pseudospectrum subsets $\Delta_{\epsilon}($.$) , where \Delta_{\epsilon}($.$) stands for any one of the$ spectral functions $\sigma_{\epsilon}(),. \sigma_{\epsilon}($.$) and \sigma_{\epsilon}^{r}$ (.) for some $\epsilon>0$. We also characterize the additive (resp. non-linear) maps from $B(X)$ onto $B(Y)$ preserving the pseudospectrum $\sigma_{\epsilon}($.$) of generalized prod-$ ucts of operators for some $\epsilon>0$ (resp. for every $\epsilon>0)$.

Keywords: Additive maps; Pseudospectrum preservers; Generalized products.

MSC (2020): Primary 47B49; Secondary 47B48, 47A10.

\section{Cite this article as (IEEE citation style):}

M. Ech-Chérif El Kettani and A. Lahssaini "On the pseudospectrum preservers", Proyecciones (Antofagasta, On line), vol. 39, no. 6, pp. 1457-1469, Dec. 2020, doi: 10.22199/issn.07176279-2020-06-0089.

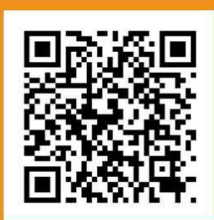

Article copyright: (C) 2020 Mustapha Ech-Chérif El Kettani and Aziz Lahssaini. This is an open access article distributed under the terms of the Creative Commons License, which permits unrestricted use and distribution provided the original author and source are credited. 


\section{Introduction}

Over the last decades there has been a considerable interest in the so called linear preserver problems, see for example $[2,5,8,9,10,12]$. The objective is to study additive or linear maps between two Banach algebras preserving or compressing a given class of elements of algebras. The most famous problem is Kaplansky's problem asking whether bijective unital linear maps between semi-simple Banach algebras preserving invertibility in both directions are Jordan isomorphisms. Note that the Kaplansky's problem mainly asks for characterizing additive maps that preserve invert- ibility. This is a much more difficult task than that for linear maps. Many mathematicians were interested to study certain quantities related on or extended the concept of spectrum. In [9], Krishna and Kulkarni gave sev- eral results about linear maps preserving pseudospectrum and condition spectrum. From what they proven, if a linear onto map preserve the pseu- dospectrum between two Banach algebras, then it preserves the spectrum. Later in [12], Ragoubi define the left pseudospectrum and the right pseu-dospectrum. Based on [9] she gives some similar results for linear maps preserving left (or right) pseudospectrum. In this paper we will prove some similar result for additive maps compressing the pseudospecturm, the left pseudospectrum or the right pseudospectrum. Also we will give some re- sults about maps preserving the pseudospectrum of generalized product. Our arguments are influenced by ideas from $[8,9,12]$.

Let $\mathcal{A}$ be a complex Banach algebra with unit 1 . We shall identify $\lambda .1$ with $\lambda$. An element $a \in \mathcal{A}$ is said to be left invertible if there exists $b \in \mathcal{A}$ such that $b a=1$, while it is said right invertible if there exists $b \in \mathcal{A}$ such that $a b=1$. An invertible element of $\mathcal{A}$ is a right and left invertible element of $\mathcal{A}$. We denote by $\operatorname{Inv}(\mathcal{A}), \operatorname{Inv}^{l}(\mathcal{A})$ and $\operatorname{Inv}^{r}(\mathcal{A})$ the sets of all invertible, left invertible and right invertible elements of $\mathcal{A}$, respectively.

Let $a \in \mathcal{A}$, the left spectrum, the right spectrum and the spectrum of $a$ are defined respectively as follow:

$$
\begin{gathered}
\sigma^{l}(a)=\left\{\lambda \in \mathbf{C}: a-\lambda \notin \operatorname{Inv}^{l}(\mathcal{A})\right\} \\
\sigma^{r}(a)=\left\{\lambda \in \mathbf{C}: a-\lambda \notin \operatorname{Inv}^{r}(\mathcal{A})\right\}
\end{gathered}
$$

and 


$$
\sigma(a)=\{\lambda \in \mathbf{C}: a-\lambda \notin \operatorname{Inv}(\mathcal{A})\} .
$$

Also the left pseudospectrum, the right pseudospectrum and the pseudospectrum of $a$ are defined respectively as follow:

$$
\begin{gathered}
\sigma_{\epsilon}^{l}(a)=\left\{\lambda \in \mathbf{C}: \inf \{\|b\|: \mathrm{b} \text { a left inverse of } a-\lambda\} \geq \frac{1}{\epsilon}\right\} \\
\sigma_{\epsilon}^{r}(a)=\left\{\lambda \in \mathbf{C}: \inf \{\|b\|: \mathrm{b} \text { a right inverse of } a-\lambda\} \geq \frac{1}{\epsilon}\right\}
\end{gathered}
$$

and

$$
\sigma_{\epsilon}(a)=\left\{\lambda \in \mathbf{C}:\left\|(a-\lambda)^{-1}\right\| \geq \frac{1}{\epsilon}\right\}
$$

with the convention $\inf \{\|b\|: b$ a left inverse of $a-\lambda\}=\infty, \inf \{\|b\|$ : $b$ a right inverse of $a-\lambda\}=\infty$ and $\left\|(a-\lambda)^{-1}\right\|=\infty$ when $a-\lambda$ is not left invertible, is not right invertible or is not invertible respectively. For more informations about this subsets we can see $[9,12,14]$.

It is clear that for all $a \in \mathcal{A}$, we have

$$
\sigma^{l}(a) \subseteq \sigma_{\epsilon}^{l}(a) \subseteq \sigma_{\epsilon}(a),
$$

and

$$
\sigma^{r}(a) \subseteq \sigma_{\epsilon}^{r}(a) \subseteq \sigma_{\epsilon}(a) .
$$

In particular $\sigma_{\epsilon}^{l}(a)$ and $\sigma_{\epsilon}^{r}(a)$ are non empty sets.

Throughout this paper, $X$ and $Y$ denote a complex Banach spaces, and $\mathcal{B}(X, Y)$ denotes the space of all bounded linear maps from $X$ into $Y$. As usual, when $X=Y$, we simply write $\mathcal{B}(X)$ instead of $\mathcal{B}(X, X)$. We denote by $T^{*}$ the operators adjoint of $T$. In the sequel $\Delta_{\epsilon}($.$\left.) (resp. \Delta().\right)$ stands for any one of the spectral functions $\sigma_{\epsilon}(),. \sigma_{\epsilon}^{l}($.$) or \sigma_{\epsilon}^{r}($.$) (resp. \sigma(),. \sigma^{l}($.$) or$ $\left.\sigma^{r}().\right)$.

\section{Preliminaries}

In this section, we collect some lemmas that will be used in the proof of our main results. We begin by a useful property of the pseudospectrum. 
Lemma 2.1. ([11, Theorem 2.3]) Let $X$ be a complex Banach space, $T$ $\in \mathcal{B}(X)$ and $\epsilon>0$. Then we have

$$
\sigma_{\epsilon}(\lambda T)=\lambda \sigma_{\frac{\epsilon}{|\lambda|}}(T), \text { for all } \lambda \in \mathbf{C} \backslash\{0\} .
$$

The second result due a Skhiri [13] gives necessary and sufficient con- dition for an operator to be an isometry multiplied by a scalar. A simple and shorter proof was given by Bourhim in ([4, Lemme 1.4])

Lemma 2.2. For a bijective mapping $A \in \mathcal{B}(X, Y)$, the following are equivalent:

1. $\left\|A T A^{-1}\right\|=\|T\|$ for all invertible operators $T \in \mathcal{B}(X)$.

2. $\left\|A T A^{-1}\right\| \leq\|T\|$ for all invertible operators $T \in \mathcal{B}(X)$.

3. $\left\|A T A^{-1}\right\| \geq\|T\|$ for all invertible operators $T \in \mathcal{B}(X)$.

4. $A$ is an isometry multiplied by a scalar.

We close this section by giving sufficient conditions for a map from $\mathcal{B}(X)$ into $\mathcal{B}(Y)$ to preserve $\Delta_{\epsilon}($.$) . Hence in what follows we will consider$ only the necessary conditions.

Lemma 2.3. ([9, Theorem 3.1 and Corollary 3.7]) Let $\phi$ be a map from $\mathcal{B}(X)$ into $\mathcal{B}(Y)$, if $\phi$ is

- linear;

- bijective;

- isometry.

Then $\phi$ preserve the $\Delta_{\epsilon}($.$) .$

\section{Additive maps compressing pseudospectrum and certain subsets}

We begin this section by establishing more lemmas needed for the proof of our main results. The first one, proved in [8, Corollary 9], gives a characterization of additive maps that compress some spectral functions.

Lemma 3.1. Let $X$ be a complex Banach space such that $\operatorname{dim}(X)=\infty$. If $\phi: \mathcal{B}(X) \longrightarrow \mathcal{B}(Y)$ is an additive surjection, which does not annihilate all rank-one idempotents, satisfies $\Delta(\phi(S)) \subseteq \Delta(S)$ for each $S \in \mathcal{B}(X)$, then $\phi$ is either an isomorphism or an anti-isomorphism. More precisely, either 
1. there exists a bounded linear bijection $A: X \longrightarrow Y$ such that $\phi(S)=$ $A S A^{-1}$, for all operator $S \in \mathcal{B}(X)$; or

2. both $X$ and $Y$ are reflexive, and there exists a bounded linear bijection $A: X^{*} \longrightarrow Y$ such that $\phi(S)=A S^{*} A^{-1}$, for all operator $S \in \mathcal{B}(X)$.

The second one proved by the some authors gives a characterization of additive maps that expand the spectral functions.

Lemma 3.2. ([8, Corollary 8]) Let $X$ be a complex Banach space such that $\operatorname{dim}(X)=\infty$. If $\phi: \mathcal{B}(X) \longrightarrow \mathcal{B}(Y)$ is an additive surjection, satisfies $\Delta(\phi(S)) \supseteq \Delta(S)$, for each $S \in \mathcal{B}(X)$. Then $\phi$ is either an isomorphism or an anti-isomorphism.

For the case of operators on Hilbert spaces operator cases they obtained the following result.

Lemma 3.3. ([8, Corollary 10]) Let $H$ and $K$ be infinite dimensional complex Hilbert spaces, and let $\phi: \mathcal{B}(H) \longrightarrow \mathcal{B}(K)$ be an additive surjection, which does not annihilate all rank-one idempotents. If $\Delta(\phi(S)) \subseteq$ $\Delta(S)$ holds for each $S \in \mathcal{B}(H)$, then either

1. there exists a bounded invertible linear operator $A: H \longrightarrow K$ such that $\phi(S)=A S A^{-1} \quad S \in \mathcal{B}(H)$; or

2. there exists a bounded invertible linear operator $A: H \longrightarrow K$ such that $\phi(S)=A S^{t r} A^{-1} \quad S \in \mathcal{B}(H)$, here $S^{t r}$ stands for the transpose of $S$ with respect to an arbitrarily fixed orthonormal base of $H$. If $\Delta($.$) takes any one of \sigma^{l}($.$) and \sigma^{r}($.$) , then the last case can not occur.$

The following is our main result for this section.

Theorem 3.4. Let $\epsilon>0$ and suppose that $\phi: \mathcal{B}(X) \longrightarrow \mathcal{B}(Y)$ is an additive surjection, which does not annihilate all rank-one idempotents. If, for each $S \in \mathcal{B}(X), \Delta_{\epsilon}(\phi(S)) \subseteq \Delta_{\epsilon}(S)$, then $\phi$ is either an isomorphism or an anti-isomorphism. More precisely, either

1. there exists an isometry $A: X \longrightarrow Y$ such that $\phi(S)=A S A^{-1}$, for all operator $S \in \mathcal{B}(X)$; or

2. both $X$ and $Y$ are reflexive, and there exists an isometry $A: X^{*} \longrightarrow$ $Y$ such that $\phi(S)=A S^{*} A^{-1}$, for all operator $S \in \mathcal{B}(X)$. 
Proof. Assume that $\Delta_{\epsilon}()=.\sigma_{\epsilon}($.$) , we will proceed by two steps.$

Step 1. $\phi$ compress the spectra of elements. Then $\phi$ takes one of the two forms in Lemma 3.1.

Suppose that $\sigma_{\epsilon}(\phi(S))=\sigma\left({ }_{\epsilon} S\right)$, for all $S \in \mathcal{B}(X)$. Let $S \in \mathcal{B}(X)$ and $\lambda \notin \sigma(S)$. Choose an integer $n$ such that $n>\epsilon\left\|(\lambda-S)^{-1}\right\|$. Then

$$
\left\|(n(\lambda-S))^{-1}\right\|<\frac{1}{\epsilon} .
$$

Thus

$$
n \lambda \notin \sigma_{\epsilon}(n S) \supseteq \sigma_{\epsilon}(\phi(n S)) \supseteq \sigma(\phi(n S))=n \sigma(\phi(S))
$$

so

$$
\lambda \notin \sigma(\phi(S))
$$

Therefore

$$
\sigma(\phi(S)) \subseteq \sigma(S)
$$

By lemma $3.1 \phi$ takes one of the two forms:

1. There exists a bounded linear bijection $A: X \longrightarrow Y$ such that $\phi(S)=$ $A S A^{-1}$, for all operator $S \in \mathcal{B}(X)$.

2. There exists a bounded linear bijection $A: X^{*} \longrightarrow Y$ such that $\phi(S)=A S^{*} A^{-1}$, for all operator $S \in \mathcal{B}(X)$. The last case may occur only if $X$ and $Y$ are reflexive.

Step 2. $\phi$ takes the desired forms.

To do so, it suffices to prove that $\phi$ preserves norms of all invertible elements of $\mathcal{B}(X)$ and applied Lemma 2.2. Indeed suppose there exist an operator invertible $S \in \mathcal{B}(X)$ such $\left\|S^{-1}\right\|<\left\|\phi\left(S^{-1}\right)\right\|$. According to the first step $\phi$ is $\mathbf{R}$-linear. Then we can choose a reel $t>0$ such that

$$
\epsilon\left\|S^{-1}\right\|<t \leq \epsilon\left\|\phi\left(S^{-1}\right)\right\|=\epsilon\left\|(\phi(S))^{-1}\right\| .
$$

Then

$$
\left\|(t S)^{-1}\right\|<\frac{1}{\epsilon}
$$

Thus $0 \notin \sigma_{\epsilon}(t S)$. But

$$
\left\|(\phi(t S))^{-1}\right\| \geq \frac{1}{\epsilon}
$$


implies $0 \in \sigma_{\epsilon}(\phi(t S))$. This is a contradiction. Similarly we can prove the result if $\Delta_{\epsilon}($.$) takes any one of the other sets, by proceeding like the proof$ of [12, Theorem 3.2].

Applying Theorem 3.4, we can give a characterization of additive maps that expand $\Delta_{\epsilon}($.$) .$

Corollary 3.5. Let $\epsilon>0$ and suppose that $\phi: \mathcal{B}(X) \longrightarrow \mathcal{B}(Y)$ is an additive surjection. If, for each $S \in \mathcal{B}(X), \Delta_{\epsilon}(\phi(S)) \supseteq \Delta_{\epsilon}(S)$, then $\phi$ is either an isomorphism or an anti-isomorphism.

Proof. Like the first step in the previous proof we can show that $\phi$ expend the spectra of elements. Hence by Lemma 3.2, $\phi$ takes the desired forms.

Remark 3.6. 1. It is obvious that if $\phi$ is bijective or it preserve $\Delta_{\epsilon}($.$) ,$ then $\phi$ does not annihilate all rank-one idempotents. So if we assume that $\phi$ is bijective or preserves $\Delta_{\epsilon}($.$) , we can drop this hypothesis.$

2. If $\phi$ preserves $\Delta_{\epsilon}($.$) we can drop condition on dimension of X$ in Theorem 3.4. We can proceed as the proof of [8, Corollary 3.16].

For the case of operators on Hilbert space, the statement of theorem 3.4 and lemma 3.3 need be slightly modified in an obvious way. We illustrate it by the following special consequence.

Corollary 3.7. Let $H$ and $K$ be infinite dimensional complex Hilbert spaces, and let $\phi: \mathcal{B}(H) \longrightarrow \mathcal{B}(K)$ be an additive surjection, which does not annihilate all rank-one idempotents. If $\Delta_{\epsilon}(\phi(S)) \subseteq \Delta_{\epsilon}(S)$ holds for each $S \in \mathcal{B}(H)$, then either

1. there exists an isometry $A: H \longrightarrow K$ such that $\phi(S)=A S A^{-1}$ for all operator $S \in \mathcal{B}(H)$; or

2. there exists an isometry $A: H \longrightarrow K$ such that $\phi(S)=A S^{t r} A^{-1}$ for all operator $S \in \mathcal{B}(H)$, here $S^{\text {tr }}$ stands for the transpose of $S$ with respect to an arbitrarily fixed orthonormal base of $H$. If $\Delta_{\epsilon}($.$) takes$ any one of $\sigma_{\epsilon}^{l}($.$) and \sigma_{\epsilon}^{r}($.$) , then the last case (2) can not occur.$ 


\section{Maps preserving pseudospectrum of generalized product}

In what follows, let $m$ be an integer and fix a positive integer $k \geq 2$ and a finite sequence $\left(i_{1}, i_{2}, \ldots, i_{m}\right)$ such that $\left\{i_{1}, i_{2}, \ldots, i_{m}\right\}=\{1,2, \ldots, k\}$ and there is an $i_{p}$ not equal to $i_{q}$ for all other $q$. We define the generalized product $T_{1} \circ T_{2} \circ \ldots \circ T_{k}$, for operators $T_{1}, \ldots, T_{k} \in \mathcal{B}(X)$ by

$$
T_{1} \circ T_{2} \circ \ldots \circ T_{k}=T_{i_{1}} \ldots T_{i_{m}},
$$

or

$$
T_{1} \circ T_{2} \circ \ldots \circ T_{k}=T_{i_{1}} \ldots T_{i_{m}}+T_{i_{m}} \ldots T_{i_{1}} .
$$

The usual product $T S$, the triple product $T S T$ and the Jordan product $T S+S T$ are a particular cases of this products. Note that maps preserving certain spectral quantities of the generalized product has been considered by many authors; see for instance $[1,3]$.

We will say that $\phi$ satisfies the condition $(\mathbf{P})$ if the the range of $\phi$ contain all operators of rank at most two if, $T_{1} \circ T_{2} \circ \ldots \circ T_{k}=T_{i_{1}} \ldots T_{i_{m}}$, or the range contain all operators of rank at most three if $T_{1} \circ T_{2} \circ \ldots \circ T_{k}=$ $T_{i_{1}} \ldots T_{i_{m}}+T_{i_{m}} \ldots T_{i_{1}}$.

Before stating the main results for this section, we will give a characterisation of maps preserving the spectrum of generalized product.

Lemma 4.1. ([6,7]) Let $X$ and $Y$ be a complex Banach spaces. If $\phi$ : $\mathcal{B}(X) \longrightarrow \mathcal{B}(Y)$ is a map satisfies the condition $(\mathbf{P})$ and $\sigma\left(\phi\left(T_{1}\right) \circ \phi\left(T_{2}\right) \circ\right.$ $\left.\ldots \circ \phi\left(T_{k}\right)\right)=\sigma\left(T_{1} \circ T_{2} \circ \ldots \circ T_{k}\right)$ for all $T_{1}, \ldots, T_{k} \in \mathcal{B}(X)$. Then one of the following conditions holds.

1. There exist a scalar $\lambda$ with $\lambda^{m}=1$ and an invertible operator $A$ : $X \longrightarrow Y$ such that $\phi(T)=\lambda A T A^{-1}$, for all operator $T \in \mathcal{B}(X)$.

2. Both $X$ and $Y$ are reflexive, and there exist a scalar $\lambda$ with $\lambda^{m}=1$ and an invertible operator $A: X^{*} \longrightarrow Y$ such that $\phi(T)=\lambda A T^{*} A^{-1}$, for all operator $T \in \mathcal{B}(X)$.

The first result characterize additive maps preserving pseudospectrum of generalized product for some $\epsilon>0$.

Theorem 4.2. Let $X$ and $Y$ be a complex Banach spaces and $\epsilon>0$. If $\phi: \mathcal{B}(X) \longrightarrow \mathcal{B}(Y)$ is an additive map satisfies the condition $(\mathbf{P})$ and $\sigma_{\epsilon}\left(\phi\left(T_{1}\right) \circ \phi\left(T_{2}\right) \circ \ldots \circ \phi\left(T_{k}\right)\right)=\sigma_{\epsilon}\left(T_{1} \circ T_{2} \circ \ldots \circ T_{k}\right)$ for all $T_{1}, \ldots, T_{k} \in \mathcal{B}(X)$. Then one of the following conditions holds. 
1. There exist a scalar $\lambda$ with $\lambda^{m}=1$ and an isometry $A: X \longrightarrow Y$ such that $\phi(T)=\lambda A T A^{-1}$, for all operator $T \in \mathcal{B}(X)$.

2. Both $X$ and $Y$ are reflexive, and there exist a scalar $\lambda$ with $\lambda^{m}=1$ and an isometry $A: X^{*} \longrightarrow Y$ such that $\phi(T)=\lambda A T^{*} A^{-1}$, for all operator $T \in \mathcal{B}(X)$.

Proof. First we will show that $\phi$ preserve the spectra of generalized product of elements. Indeed, let $\lambda \notin \sigma\left(T_{1} \circ T_{2} \circ \ldots \circ T_{k}\right)$. Choose $n>\epsilon \|$ $\left(\lambda-T_{1} \circ T_{2} \circ \ldots \circ T_{k}\right)^{-1} \|$. Then

$$
\left\|\left(n\left(\lambda-T_{1} \circ T_{2} \circ \ldots \circ T_{k}\right)\right)^{-1}\right\|<\frac{1}{\epsilon} .
$$

Thus

$$
\begin{aligned}
n \lambda \notin \sigma_{\epsilon}\left(n T_{1} \circ T_{2} \circ \ldots \circ T_{k}\right) & =\sigma_{\epsilon}\left(\phi\left(n T_{1}\right) \circ \phi\left(T_{2}\right) \circ \ldots \circ \phi\left(T_{k}\right)\right) \\
& \supseteq \sigma\left(\phi\left(n T_{1}\right) \circ \phi\left(T_{2}\right) \circ \ldots \circ \phi\left(T_{k}\right)\right) \\
& =n \sigma\left(\phi\left(T_{1}\right) \circ \phi\left(T_{2}\right) \circ \ldots \circ \phi\left(T_{k}\right)\right)
\end{aligned}
$$

SO

$$
\lambda \notin \sigma\left(\phi\left(T_{1}\right) \circ \phi\left(T_{2}\right) \circ \ldots \circ \phi\left(T_{k}\right)\right) .
$$

Therefore

$$
\sigma\left(\phi\left(T_{1}\right) \circ \phi\left(T_{2}\right) \circ \ldots \circ \phi\left(T_{k}\right)\right) \subseteq \sigma\left(\phi\left(T_{1}\right) \circ \phi\left(T_{2}\right) \circ \ldots \circ \phi\left(T_{k}\right)\right) .
$$

In a similar way we can prove the other inclusion. Hence we get

$$
\sigma\left(\phi\left(T_{1}\right) \circ \phi\left(T_{2}\right) \circ \ldots \circ \phi\left(T_{k}\right)\right)=\sigma\left(\phi\left(T_{1}\right) \circ \phi\left(T_{2}\right) \circ \ldots \circ \phi\left(T_{k}\right)\right) .
$$

Now by the previous lemma $\phi$ takes one of the two forms:

1. There exist a scalar $\lambda$ with $\lambda^{m}=1$ and an invertible operator $A$ : $X \longrightarrow Y$ such that $\phi(T)=\lambda A T A^{-1}$, for all operator $T \in \mathcal{B}(X)$.

2. Both $X$ and $Y$ are reflexive, and there exist a scalar $\lambda$ with $\lambda^{m}=1$ and an invertible operator $A: X^{*} \longrightarrow Y$ such that $\phi(T)=\lambda A T^{*} A^{-1}$, for all operator $T \in \mathcal{B}(X)$. 
Finally, let us prove that $\left\|A S A^{-1}\right\| \leq\|S\|$ of all invertible elements $S \in \mathcal{B}(X)$. Indeed suppose there exist an operator invertible $S \in \mathcal{B}(X)$ such $\left\|S^{-1}\right\|<\left\|A S^{-1} A^{-1}\right\|$. According to the first step $\phi$ is $\mathbf{R}$-linear and $\sigma_{\epsilon}(T)=\sigma_{\epsilon}\left(\lambda^{m-1} \phi(T)\right)=\sigma_{\epsilon}\left(A T A^{-1}\right)$ or $\sigma_{\epsilon}(T)=\sigma_{\epsilon}\left(A T^{*} A^{-1}\right)$, for every $T \in \mathcal{B}(X)$. Then we can choose $t>0$ such that

$$
\epsilon\left\|S^{-1}\right\|<t \leq \epsilon\left\|A S^{-1} A^{-1}\right\|=\epsilon\left\|\left(A S A^{-1}\right)^{-1}\right\| .
$$

Then

$$
\left\|(t S)^{-1}\right\|<\frac{1}{\epsilon}
$$

Thus $0 \notin \sigma_{\epsilon}(t S)$. But

$$
\left\|(\phi(t S))^{-1}\right\| \geq \frac{1}{\epsilon}
$$

implies $0 \in \sigma_{\epsilon}(\phi(t S))$, a contradiction. Then by Lemma $2.2 \phi$ takes the desired forms.

Next result characterize maps preserving pseudospectrum of generalized product for every $\epsilon>0$.

Theorem 4.3. Let $X$ and $Y$ be a complex Banach spaces. If $\phi: \mathcal{B}(X) \longrightarrow$ $\mathcal{B}(Y)$ is a map satisfies the condition $(\mathbf{P})$ and $\sigma_{\epsilon}\left(\phi\left(T_{1}\right) \circ \phi\left(T_{2}\right) \circ \ldots \circ \phi\left(T_{k}\right)\right)=$ $\sigma_{\epsilon}\left(T_{1} \circ T_{2} \circ \ldots \circ T_{k}\right)$ for all $T_{1}, \ldots, T_{k} \in \mathcal{B}(X)$ and every $\epsilon>0$. Then one of the following conditions holds.

1. There exist a scalar $\lambda$ with $\lambda^{m}=1$ and an isometry $A: X \longrightarrow Y$ such that $\phi(T)=\lambda A T A^{-1}$, for all operator $T \in \mathcal{B}(X)$.

2. Both $X$ and $Y$ are reflexive, and there exist a scalar $\lambda$ with $\lambda^{m}=1$ and an isometry $A: X^{*} \longrightarrow Y$ such that $\phi(T)=\lambda A T^{*} A^{-1}$, for all operator $T \in \mathcal{B}(X)$.

Proof. We will proceed like the proof of the previous theorem. First we show that $\phi$ preserve the spectra of generalized product of elements. Indeed, let $\lambda \notin \sigma\left(T_{1} \circ T_{2} \circ \ldots \circ T_{k}\right)$. Choose $n>\epsilon\left\|\left(\lambda-T_{1} \circ T_{2} \circ \ldots \circ T_{k}\right)^{-1}\right\|$. Then

$$
\left\|\left(n\left(\lambda-T_{1} \circ T_{2} \circ \ldots \circ T_{k}\right)\right)^{-1}\right\|<\frac{1}{\epsilon} .
$$

Thus 


$$
\begin{aligned}
n \lambda \notin \sigma_{\epsilon}\left(n T_{1} \circ T_{2} \circ \ldots \circ T_{k}\right) & =n \sigma_{\frac{\epsilon}{n}}\left(T_{1} \circ T_{2} \circ \ldots \circ T_{k}\right) \\
& =n \sigma_{\frac{\epsilon}{n}}\left(\phi\left(T_{1}\right) \circ \phi\left(T_{2}\right) \circ \ldots \circ \phi\left(T_{k}\right)\right) \\
& \supseteq n \sigma\left(\phi\left(T_{1}\right) \circ \phi\left(T_{2}\right) \circ \ldots \circ \phi\left(T_{k}\right)\right) .
\end{aligned}
$$

SO

$$
\lambda \notin \sigma\left(\phi\left(T_{1}\right) \circ \phi\left(T_{2}\right) \circ \ldots \circ \phi\left(T_{k}\right)\right) .
$$

Therefore

$$
\sigma\left(\phi\left(T_{1}\right) \circ \phi\left(T_{2}\right) \circ \ldots \circ \phi\left(T_{k}\right)\right) \subseteq \sigma\left(\phi\left(T_{1}\right) \circ \phi\left(T_{2}\right) \circ \ldots \circ \phi\left(T_{k}\right)\right) .
$$

In a similar way we can prove the other inclusion. Hence we get

$$
\sigma\left(\phi\left(T_{1}\right) \circ \phi\left(T_{2}\right) \circ \ldots \circ \phi\left(T_{k}\right)\right)=\sigma\left(\phi\left(T_{1}\right) \circ \phi\left(T_{2}\right) \circ \ldots \circ \phi\left(T_{k}\right)\right) .
$$

Now by the lemma $4.1 \phi$ takes one of the two forms:

1. There exist a scalar $\lambda$ with $\lambda^{m}=1$ and an invertible operator $A$ : $X \longrightarrow Y$ such that $\phi(T)=\lambda A T A^{-1}$, for all operator $T \in \mathcal{B}(X)$.

2. Both $X$ and $Y$ are reflexive, and there exist a scalar $\lambda$ with $\lambda^{m}=1$ and an invertible operator $A: X^{*} \longrightarrow Y$ such that $\phi(T)=\lambda A T^{*} A^{-1}$, for all operator $T \in \mathcal{B}(X)$.

Finally like the previous proof we prove that $\left\|A S A^{-1}\right\| \leq\|S\|$ for all invertible elements $S \in \mathcal{B}(X)$. Then by Lemma $2.2 \phi$ takes the desired forms.

Question 4.4. Can we change the condition "for every $\epsilon>0$ " in Theorem 4.3 by "some $\epsilon>0$ ".

\section{References}

[1] Z. E. A. Abdelali, A. Achchi, and R. Marzouki, "Maps preserving the local spectral radius zero of generalized product of operators", Linear and multilinear algebra, vol. 67, no. 10, pp. 2021-2029, Jul. 2018, doi: 10.1080/03081087.2018.1479371 
[2] B. Aupetit, "Spectrum-preserving linear mappings between Banach algebras or Jordan-Banach algebras", Journal of the London mathematical society, vol. 62, no. 3, pp. 917-924, Dec. 2000, doi: 10.1112/ S0024610700001514

[3] H. Benbouziane, Y. Bouramdane, and M. Ech-Chérif El Kettani, "Maps preserving local spectral subspaces of generalised product of operators", Rendiconti del Circolo Matematico di Palermo Series 2, vol. 69, no. 3, pp. 1033-1042, Dec. 2019, doi: 10.1007/s12215-01900453-w

[4] A. Bourhim, "Additive maps preserving the reduced minimum modulus of Banach space operators", Journal of operator theory, vol. 67, no. 1, pp. 279-288, 2012. [On line]. Available: https:/ / bit.ly/ 34CA0n1

[5] M. Ech-Chérif El Kettani and H. Benbouziane, "Additive maps preserving operators of inner local spectral radius zero", Rendiconti del Circolo Matematico di Palermo, vol. 63, no. 2, pp. 311-316, Aug. 2014, doi: 10.1007/ s12215-014-0160-z

[6] J. Hou, C.-K. Li, and N.-C. Wong, "Jordan isomorphisms and maps preserving spectra of certain operator products", Studia mathematica, vol. 184, no. 1, pp. 31-47, 2008, doi: 10.4064/ sm184-1-2

[7] J. Hou, C.-K. Li, and N.-C. Wong, "Maps preserving the spectrum of generalized Jordan product of operators", Linear algebra and its applications, vol. 432, no. 4, pp. 1049-1069, Feb. 2010, doi: 10.1016/j.laa.2009.10.018

[8] J. Hou and L. Huang, "Additive maps between standard operator algebras compressing certain spectral functions", Acta mathematica sinica, english series, vol. 24, no. 12, pp. 2041-2048, Dec. 2008., Dec. 2008, doi: 10.1007/ s10114-008-6428-5

[9] G. K. Kumar and S. H. Kulkarni, "Linear maps preserving pseudospectrum and condition spectrum", Banach journal of mathematical analysis, vol. 6, no. 1, pp. 45-60, 2012, doi: 10.15352/ bjma/ 1337014664

[10] B. Kuzma, "Additive spectrum compressors", Journal of mathematical analysis and applications, vol. 304, no. 1, pp. 13-21, Apr. 2005, doi: 10.1016/j.jmaa.2004.09.004

[11] A. Krishnan and S. H. Kulkarni, "Pseudo spectrum of element in Banach algebra”, Operators and matrices, vol. 11, no. 1, pp. 263-287, Mar. 2017, doi: 10.7153/ oam-11-18 
[12] S. Ragoubi, "On linear maps preserving certain pseudospectrum and condition spectrum subsets", Advances in operator theory, vol. 3, no. 2, pp. 423-432, 2018, doi: 10.15352/ AOT.1705-1159

[13] H. Skhiri, "Reduced minimum modulus preserving in Banach space", Integral equations and operator theory, vol. 62, no. 1, pp. 137-148, Sep. 2008, doi: 10.1007/ s00020-008-1612-7.

[14] L. N. Trefethen and M. Embree, Spectra and pseudospectra: the behavior of nonnormal matrices and operators. Princeton, NJ: Princeton University Press, 2005. 